\title{
Improving preparedness for the next flu pandemic
}

\author{
Pandemic influenza remains the single greatest threat to global heath security. Efforts to increase our preparedness, by \\ improving predictions of viral emergence, spread and disease severity, by targeting reduced transmission and improved \\ vaccination and by mitigating health impacts in low- and middle-income countries, should receive renewed urgency.
}

\author{
Peter Horby
}

G iven its potential to cause an acute global health crisis with many millions of deaths, pandemic influenza can rightly be considered the greatest single threat to global health security. Yet despite this threat, influenza has recently been eclipsed in the popular and scientific consciousness by less common infections, such as those caused by Ebola and Zika viruses. The centenary of the 1918 influenza pandemic, which is estimated to have killed 50 million people, is an opportune time to remind ourselves that the greatest risks often lay in the mundane. Much like we need reminding that driving is far more dangerous than flying, familiarity can breed contempt.

The appearance of a human influenza pandemic depends on the emergence of a novel virus that can readily infect and transmit between people. The mostly likely source of a pandemic virus is the pool of influenza viruses that infect animals such as wild birds (the natural reservoir of influenza A viruses), domestic poultry and pigs. The scale of pig and poultry farming has increased massively over the past 50 years, with the estimated global number of pigs and chickens having increased roughly twofold and fivefold, respectively, from the early 1960s until now (from 400 million to around 1 billion pigs; and 4 billion to 20 billion chickens) ${ }^{1}$. Swine influenza is endemic in pig populations, with co-circulation of multiple subtypes and the intermittent introduction of new strains from avian or human sources, whilst poultry populations are affected by an increasing variety of influenza viruses. Human infections with a diverse range of zoonotic influenza viruses are now being detected (Fig. 1), some of which are associated with a high case fatality rate, and some of which have mutations that confer resistance to the major classes of influenza antiviral drugs.

Although it is hard to be sure as surveillance and genetic sequencing capabilities have been increasing, we seem to be experiencing a notable increase in the genetic exchange and diversification of animal influenza viruses ${ }^{2}$. Determining which of these viruses will cause the next pandemic, when it will happen and how bad it will be remains incredibly challenging. However, there are warning signs to be heeded and areas in which our preparedness could be strengthened to make sure that we are best placed to identify and swiftly confront the next influenza pandemic.

\section{Improving predictions}

Influenza is probably one of the most studied viruses, yet fundamental gaps exist in our ability to predict the transmissibility and virulence of novel influenza viruses. The recent lifting by the US National Institutes of Health of their moratorium ${ }^{3}$ on funding of so-called 'gain-of-function' experiments (laboratory experiments where viruses are genetically altered to assess the effect on properties such as transmissibility and virulence) is good news, as it allows us to explore the limits of evolution and the genotypic predictors of phenotype $\mathrm{e}^{4}$. However, there are limits to the predictive value of laboratory experiments and animal models, and when a new pandemic virus does emerge, which inevitably it will, the most important measure will be the disease severity per infected person. This information is crucial for understanding the potential impact of the pandemic and appropriately calibrating the political and public health response. Estimating the severity per infected person requires robust and real-time data on the number of people infected and the proportion within this group that develop severe disease $\mathrm{e}^{5}$. Such data are surprisingly hard to gather and interpret because care-seeking and care-giving behaviour can change as awareness of a pandemic and pressures on healthcare change. This is an area that requires investment in methodologies and tools for gathering the necessary input data, including potential modifying factors such as care-seeking behaviour, and feeding that data into analytic frameworks for estimating the severity per infected person.

Mathematical models of disease transmission have become a routine tool for evaluating and predicting the behaviour of epidemics. These models are attractive to public health officials as they provide a quantitative answer to many questions, but most importantly to: "how bad is this and how bad might it get?" A promising approach to even faster and more reliable characterization of outbreaks is 'modeldriven data collection', which tells us "how much data to collect and when to collect it" in order to improve the predictive power of the models and to maximize the efficiency of data collection ${ }^{6}$. This concept should be tested by developing and piloting modeldriven data collection systems for seasonal or zoonotic influenza, to see what and how much data are needed to provide estimates of the severity per infected person that are sufficiently reliable to be actionable.

\section{Reducing transmission}

The world population is around four times the size it was in 1918, and the mobility of this population is massively increased ${ }^{4}$. If a potential pandemic influenza virus acquires the ability to readily transmit between humans, it will spread with alarming speed and essentially become unstoppable. Aeroplane transportation is the greatest facilitator of rapid global spread ${ }^{7}$, yet airport screening has limited ability to prevent importation and at best can lead to a short delay (less than two weeks) in the onset of local transmission ${ }^{8,9}$. At a national level, measures to limit local transmission, such as school closure, can have some effect in reducing community transmission and can mitigate pressures on the healthcare system by reducing peak incidence. The effect is, however, dependent on timing and coordination and therefore requires access to real-time surveillance data, such as absenteeism ${ }^{10,11}$. The simultaneous use of face 


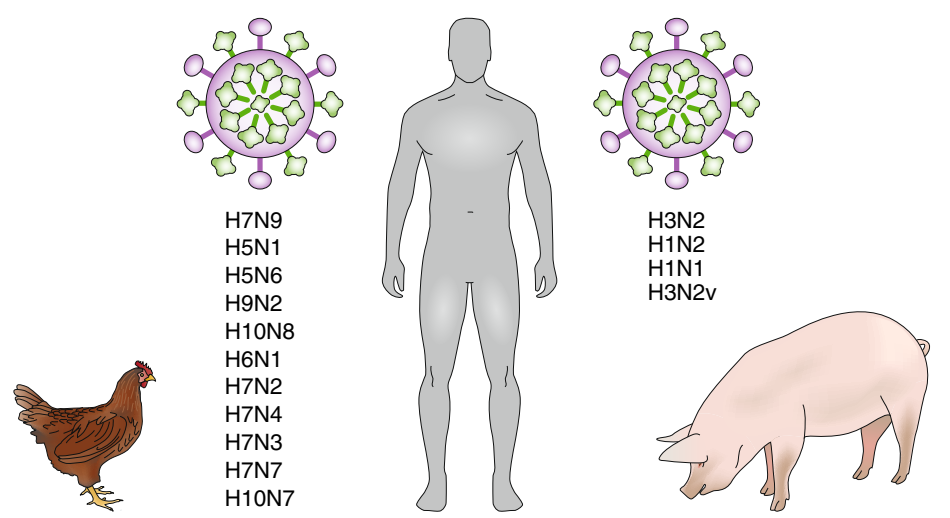

Fig. 1 | Detected human infections with avian and swine influenza viruses. $H$ denotes the haemagglutinin surface protein subtype (of which 18 are currently identified), whilst $\mathrm{N}$ denotes the neuraminidase surface protein subtype (of which 11 are currently identified).

masks and hand hygiene together, targeting both aerosol and contact transmission, can reduce spread under laboratory conditions, although the effectiveness of these measures as a community-wide intervention is unproven $^{12}$. Whilst neuraminidase inhibitors (NAIs) have been demonstrated to be effective in reducing symptomatic influenza and intra-household transmission when used prophylactically, there are no data on the effectiveness or cost effectiveness of NAI use in reducing community-wide transmission ${ }^{13}$. Therefore, the stockpiling of NAIs by individual countries could permit prophylactic use that may reduce the local impact of a pandemic, but at the global level NAIs are not likely to have a significant role in reducing pandemic influenza transmission.

\section{Reducing the health impact}

Around half of the world's population lacks full access to essential health services ${ }^{14}$. It is therefore not surprising that the burden of influenza is greater in low- and middleincome countries (LMICs) than in highincome countries. In the 2009 pandemic, the estimated death rate in Africa was 2-4 times that of other regions ${ }^{15}$, whilst mortality rates for influenza-associated acute lower respiratory tract infection in children younger than 5 years are three times higher in low-income countries than in high-income countries ${ }^{16}$. The influenzaassociated death rate in the elderly is also likely to be substantially increased in LMICs compared to higher-income countries ${ }^{17}$. This burden of influenza in LMICs is often unrecognized, particularly in rural areas $^{18,19}$. Any assessment of our ability to mitigate the health impact of the next influenza pandemic must consider what will be available for the large and vulnerable populations living in LMICs.
The therapeutic efficacy of NAIs is a matter of some debate, but can probably be summarized as a proven but modest effect on the duration of symptoms in patients with mild influenza, and a probable but unproven small reduction in severe outcomes such as pneumonia and death. Given the weak evidence of an impact on severe outcomes, the NAI oseltamivir has recently been 'downgraded' to the complementary part of the WHO Essential Medicines List, only to be used for critically ill patients hospitalized with influenza ${ }^{20}$. It is, therefore, hard to see NAIs having a significant global impact on the rate of hospitalization or death in any future pandemic. In addition to the weak evidence of an impact on severe outcomes, the existence of viral genetic variants that confer considerable resistance to NAIs make it clear that new antivirals for influenza are needed. There are candidates in clinical development but progress is slow, and companies have favoured evaluating new candidate drugs in patients with uncomplicated influenza, since this is the bigger market. What is needed is a large influenza clinical trials network that can evaluate multiple candidates and combination therapies (including antiviral combinations and host-directed therapies) in an adaptive platform trial, as has been achieved for cancer, for example with the I-SPY2 platform trial ${ }^{21}$.

However, at this time, interventions other than antivirals can probably offer more health benefit globally. The two simple interventions that can save lives are antibiotics and oxygen. Secondary bacterial infections, largely due to Streptococcus pneumoniae, are thought to have made a substantial contribution to influenzaassociated pneumonia and death in 1918 $\left(\right.$ ref. $\left.{ }^{22}\right)$. Despite the widespread availability of antibiotics, only around half of children with pneumonia in LMICs receive care from a healthcare provider who can give appropriate antibiotics ${ }^{23}$. Although oxygen therapy is considered an essential medicine, reliable access to oxygen is absent in much of the world due to technical and cost challenges ${ }^{24}$. The other intervention likely to have a major impact is pneumococcal conjugate vaccines (PCVs). These vaccines have been shown to reduce the risk of influenza-associated pneumococcal disease $^{25}$, and an analysis in China suggests that routine PCV immunization prior to a pandemic would have a major impact on mortality and would be cost saving ${ }^{26}$. Globally, only $37 \%$ of children received three doses of PCV in 2015, although coverage is actually higher in low-income than highincome countries due to support from agencies such as Gavi, the Vaccine Alliance ${ }^{27}$. Effective, safe and affordable interventions, such as antibiotics, oxygen and PCVs, will save lives during an influenza pandemic and are available now. Such interventions should be made available to all.

\section{Influenza vaccination}

Whilst current influenza vaccines meet an important need and their wider use should be promoted, traditional influenza immunization approaches and the predominant egg-based production methods seriously limit their value as a tool in pandemic preparedness. There has certainly been progress in the speed of development and breadth of candidate vaccine viruses, and in the diversification and expansion of production capacity - global pandemic influenza vaccine production capacity is at its highest level ever, and may be sufficient to immunize $43 \%$ of the current global population with two doses, or $86 \%$ with one dose $\mathrm{e}^{28}$. Nevertheless, the time frame to produce and evaluate new vaccines against novel antigenic variants and to switch vaccine production is still too slow to have a substantial impact on the first wave of a pandemic. Only time will tell if the notional pandemic vaccine production capacity will ever be realized, and a pandemic vaccine will find its way into people's arms in time. Substantial efforts are ongoing to optimize the use of existing vaccine technologies and to develop novel approaches, including, most importantly, work to realize the aspiration of a broadly protective, universal influenza vaccine. These efforts are of major global importance and must continue to be supported.

\section{A renewed urgency}

If we do not keep our eye on the ball and continue to aggressively pursue improvements in pandemic influenza 
preparedness across the whole range of disciplines, including basic science, vaccinology, clinical evaluation and therapeutics, public health and health service delivery, we will be culpable of neglecting the obvious risks in favour of the more exotic and dramatic. Whilst the recently updated World Health Organization public health research agenda for influenza has some value, it does not constitute a strategic plan of action. As we have seen being recently developed for other high-threat pathogens, such as the viral haemorrhagic fevers and Middle East respiratory syndrome coronavirus, what is needed is a blueprint for action against pandemic influenza that is comprehensive, detailed, endorsed, funded and monitored.

Peter Horby ${ }^{1,2}$

${ }^{1}$ Centre for Tropical Medicine and Global Health, University of Oxford, Oxford, UK. ${ }^{2}$ International Severe Acute Respiratory and Emerging Infections
Consortium, Oxford, UK.

e-mail:peter.horby@ndm.ox.ac.uk

Published online: 25 July 2018

https://doi.org/10.1038/s41564-018-0206-7

References

1. FAOSTAT: Live Animals (FAO); http://www.fao.org/faostat/ en/\#data/QA

2. WHO. Wkly Epidemiol. Rec. 92, 129-144 (2017).

3. Notice announcing the removal of the funding pause for gain-offunction research projects. NIH (19 December 2017).

4. Pybus, O. G., Tatem, A. J. \& Lemey, P. Proc. Biol. Sci. 282, 20142878 (2015).

5. Lipsitch, M. et al. Biosecur. Bioterror. 9, 89-115 (2011).

6. Lessler, J., Edmunds, W. J., Halloran, M. E., Hollingsworth, T. D. \& Lloyd, A. L. Epidemics 10, 78-82 (2015).

7. Browne, A., Ahmad, S. S., Beck, C. R. \& Nguyen-Van-Tam, J. S. J. Travel. Med. 23, tav002 (2016).

8. Priest, P. C., Jennings, L. C., Duncan, A. R., Brunton, C. R. \& Baker, M. G. Am. J. Public Health 103, 1412-1418 (2015).

9. Cowling, B. J. et al. BMC Infect. Dis. 10, 8 (2010).

10. Fumanelli, L., Ajelli, M., Merler, S., Ferguson, N. M. \& Cauchemez, S. PLoS Comput. Biol. 12, e1004681 (2016).

11. Rashid, H. et al. Paediatr. Respir. Rev. 16, 119-126 (2015).

12. Wong, V. W., Cowling, B. J. \& Aiello, A. E. Epidemiol. Infect. 142 922-932 (2014)

13. Okoli, G. N., Otete, H. E., Beck, C. R. \& Nguyen-Van-Tam, J. S. PLoS ONE 9, el13633 (2014).
14. Universal Health Coverage (WHO); http://apps.who.int/gho cabinet/uhc.jsp

15. Dawood, F. S. et al. Lancet Infect. Dis. 12, 687-695 (2012).

16. Nair, H. et al. Lancet 378, 1917-1930 (2011).

17. Cohen, C. et al. Clin. Infect. Dis. 51, 1362-1369 (2010).

18. Yu, H. et al. Influenza Other Respir. Viruses 7 , 1350-1360 (2013).

19. Chadha, M. S. et al. PLoS ONE 8, e55918 (2013).

20. WHO Model Lists of Essential Medicines (WHO, 2017); http:// www.who.int/medicines/publications/essentialmedicines/en/

21. The I-SPY2 Trial (I-SPY); https://www.ispytrials.org/i-spyplatform/i-spy2

22. Chien, Y. W., Klugman, K. P. \& Morens, D. M. N. Engl. J. Med. 361, 2582-2583 (2009).

23. Campbell, H. et al. PLoS Med. 10, e1001421 (2013).

24. Duke, T. et al. Int. J. Tuberc. Lung Dis. 14, 1362-1368 (2010).

25. Madhi, S. A. \& Klugman, K. P., Vaccine Trialist Group. Nat. Med. 10, 811-813 (2004).

26. Caldwell, R., Roberts, C. S., An, Z., Chen, C. I. \& Wang, B. BMC Infect. Dis. 15, 284 (2015).

27. Casey, R. M. et al. MMWR Morb. Mortal Wkly Rep. 65 $1270-1273(2016)$

28. McLean, K. A., Goldin, S., Nannei, C., Sparrow, E. \& Torelli, G. Vaccine 34, 5410-5413 (2016).

Competing interests

The author declares no competing interests. 